\title{
BMJ Open Group practice impacts on patients, physicians and healthcare systems: a scoping review
}

\author{
Terry Zwiep (1) , ${ }^{1}$ San (Hilalion) Ahn, ${ }^{2}$ Jamie Brehaut, ${ }^{3}$ Fady Balaa, ${ }^{2}$ \\ Daniel I Mclsaac, ${ }^{4}$ Susan Rich, ${ }^{2}$ Tom Wallace, ${ }^{2}$ Husein Moloo ${ }^{3,5}$
}

To cite: Zwiep T, Ahn S(Hilalion), Brehaut J, et al. Group practice impacts on patients, physicians and healthcare systems: a scoping review. BMJ Open 2021;11:e041579. doi:10.1136/ bmjopen-2020-041579

- Prepublication history and additional material for this paper are available online. To view these files, please visit the journal online (http://dx.doi org/10.1136/bmjopen-2020041579).

Received 22 June 2020 Revised 13 December 2020 Accepted 29 December 2020

Check for updates

(c) Author(s) (or their employer(s)) 2021. Re-use permitted under CC BY-NC. No commercial re-use. See rights and permissions. Published by BMJ.

${ }^{1}$ Surgery, Western University, London, Ontario, Canada

${ }^{2}$ Surgery, The Ottawa Hospital, Ottawa, Ontario, Canada

${ }^{3}$ Clinical Epidemiology Program, Ottawa Hospital Research Institute, Ottawa, Ontario, Canada

${ }^{4}$ Anesthesiology and Pain Medicine, The Ottawa Hospital, Ottawa, Ontario, Canada

${ }^{5}$ Surgical Oncology, The Ottawa Hospital, Ottawa, Ontario, Canada

Correspondence to

Dr Husein Moloo;

hmolo0@toh.ca

\section{ABSTRACT}

Objective To identify the advantages and disadvantages that group practices have on patients, physicians and healthcare systems.

Study design A scoping review was performed based on the methodology proposed by Arksey and 0'Malley, and refined by Levac et al. Titles and abstracts were screened by two reviewers. A quantitative analysis was performed to assess the type, year and region of publication, as well as the population studied. A qualitative descriptive analysis was performed to identify common themes.

Study setting MEDLINE, EMBASE and Cochrane databases were searched from database inception to October 2018 for papers which assessed outcomes relevant to the research question.

Results Our search strategy returned 2408 papers and 98 were included in the final analysis. Most papers were from the USA, were surveys and assessed physician outcomes. Advantages of group practices for patients included improved satisfaction and quality of care. Studies of physicians reported improved quality of life and income, while disadvantages included increased stress due to poor interpersonal relationships. Studies of healthcare systems reported improved efficiency and better utilisation of resources.

Conclusions Group practices have many benefits for patients and physicians. However, further work needs to be done assessing patient outcomes and establishing the elements that make a group practice successful.

\section{INTRODUCTION}

Group practices have existed for over 100 years with one of the first groups set up by the Mayo brothers in the mid-1880s. ${ }^{1}$ This group was eventually transformed into a large organisation that has been recognised as a centre of excellence leading to benefits for patients and physicians. Following their success, group practices became more and more common, and currently, many physicians around the world are practising within groups or partnerships. ${ }^{2-5}$ Sizes of group practices vary dramatically, from 2 physicians to over 100 physicians, and there is no standard definition of what defines a group. Over the years many papers have been published

\section{Strengths and limitations of this study}

This was a large, comprehensive overview of group practices from many countries.

- The scoping review methodology allowed us to assess a wide variety of papers and identify key gaps in the knowledge for further study.

- Patient engagement was instrumental on focusing this review on patient outcomes and areas for improvement.

- This review was limited by language restrictions, heterogeneity of the data and possible publication bias.

on group practice formation assessing various advantages and disadvantages for patients, physicians and healthcare systems, as well as the impetus behind their development. The economic benefits of these groups and the improvements in service provision to patients are supported by the literature and has been well documented. ${ }^{36-8}$ Barriers to the formation of group practices or conflicts that can result from group practices have also been considered and often have to do with interpersonal relationships. ${ }^{9-12}$ The extent of literature spans many decades and provides an excellent overview of how group practices have evolved and the effects which they have had on patients, physicians and healthcare systems.

Patient care can be significantly altered by the formation of group practices, and it is important to consider this impact as groups are often formed for reasons that are not directly related to patient care. ${ }^{11}{ }^{13-15}$ Some of these other reasons include the benefits realised by physicians with regard to income, quality of life, satisfaction and decreased physician burnout, which is estimated to affect more than half of physicians. ${ }^{9}$ 16-19 Group practices also may be developed due to healthcare system incentives, or as a way to improve the income and efficiency of 
physicians within a given system. ${ }^{140-23}$ This again may not be directly related to patient care but may have impacts on the quality of care and its timeliness. Whatever the motivation for forming group practices, it is important to assess the effects on all involved stakeholders to ensure that this is a step in the right direction for the patients that healthcare providers are committed to serve, the well-being of physicians and the sustainability of the systems which healthcare providers work within.

There has been a paucity of literature that synthesises the knowledge published regarding group practices. A systematic review published in 2013 assessed the effectiveness of group versus solo practice among general practitioners and demonstrated a positive association between group practices and clinical processes, physicians' opinions and innovation, but did not observe any effect for patient measures. ${ }^{7}$ A recent review has also attempted to establish a definition for group practices and the overall shift towards their development. ${ }^{24}$ The objectives of this study were to review the literature for evidence that assesses the advantages and disadvantages that group practices have on patients with regard to quality of care and satisfaction; physicians with regard to team dynamics, income and satisfaction; and the financial impact on healthcare systems. A scoping review was performed as we expected to identify heterogeneous studies with a wide range of outcomes focused on patients, physicians and healthcare systems. A broad overview of the literature was desired to identify current knowledge gaps and guide further studies.

\section{METHODOLOGY}

A scoping review was performed according to the methodology proposed by Arksey and O'Malley, and refined by Levac et al. ${ }^{2526}$ The Preferred Reporting Items for Systematic Reviews and Meta-Analyses extension for scoping reviews (PRISMA-ScR) checklist was used to ensure all relevant aspects of a scoping review were included. ${ }^{27}$ The following research question was developed:

What advantages and disadvantages do group practices have for patients, physicians and healthcare systems?

A complete scoping review protocol was developed and published. ${ }^{28}$ The following stages were incorporated into this scoping review according to what is suggested by Levac et al: identifying the research question; identifying relevant studies; study selection; charting the data; collating, summarising and reporting results; and consultation. Full details on each stage can be found in the published protocol. ${ }^{28}$ Briefly, MEDLINE, EMBASE and Cochrane Central were searched from database inception to October 2018 to identify relevant studies that assess the impact of group practices on patient care, satisfaction and outcomes; physician quality of life, satisfaction and income; and healthcare system finances. There were no restrictions placed on publication date. The grey literature was not searched as originally indicated in the protocol due to an adequate number of peer-reviewed articles which met inclusion criteria from the databases. The search strategy was peer reviewed according to the formal process outlined by McGowan et al. ${ }^{29}$ The search strategy is included in online supplemental appendix A.

Three members of the research team met to perform a calibration exercise and review 10 papers to pilot the screening and full-text data extraction forms. Titles and abstracts were subsequently screened independently by two reviewers and the abstraction results from the fulltext articles were charted and verified by the same two members. Disagreements were resolved by discussion between the two reviewers as well as input from other authors of the paper. We included papers that:

- Included patients receiving, and/or clinicians providing care within any type of group practice (population).

- Assessed the advantages and/or disadvantages of group practices (concept).

We examined all papers from group practices in all areas of medicine which reported outcomes relevant to patients, clinicians or health system stakeholders (context).

Papers were excluded if they were not published in the English language.

DistillerSR (Evidence Partners, Ottawa, Canada) was used for screening and data extraction. A standardised form was created and tested on 10 papers by three members of the research team. We did not deviate from the protocol and charted authors, year of publication, country of origin, objectives, type and size of group practice, population studied (patients, physicians and so on), sample size, methods and type of study, interventions, outcomes and key findings. ${ }^{28}$ This standardised form was used throughout the study and no changes were required after it was tested.We extracted and summarised included paper characteristics including type of study, year, region of publication and the population studied. A qualitative analysis was also performed using a qualitative descriptive approach from the key findings of the selected papers. ${ }^{30}$ A coding manual was created and codes were applied to the key findings. These were refined as the study progressed and grouped into themes. This was performed in parallel by two reviewers who then met to discuss the results and corresponding themes. After a conventional content analysis, common themes were grouped by:

1. Patient care, including satisfaction and quality of care.

2. Physicians, including quality of life, competency, group dynamics, group characteristics and financial impacts.

3. Healthcare system issues relating to financial impacts.

A detailed quality assessment was not performed due to the heterogeneity of the data and the general principles of a scoping review. ${ }^{26}$ During the scoping review process, we consulted with four surgeons from other group practices to ensure that the review was comprehensive and that all relevant papers were included. These surgeons were known by the research group to be participants in group practices. 


\section{Patient and public involvement}

A patient advisor was recruited from the Department of Patient Relations as part of the research team. As practice organisation directly impacts on patients, it was essential that we had patient input into the design of the study and the analysis of the data. The patient advisor collaborated with the team and ensured that the research question and outcomes were applicable to patients and reviewed the final draft of the paper. ${ }^{31}$

\section{RESULTS}

Using the search strategy outlined in online supplemental appendix A, 2408 papers were identified. Of these, 35 were excluded as duplicates and 2373 titles and abstracts were screened. After screening, 149 full-text articles were examined and 98 met inclusion criteria. Of those excluded, 34 did not assess advantages or disadvantages of group practices, 5 papers focused on multidisciplinary groups, 2 papers were based on a previous paper and did not provide any new data, and 1 paper assessed a dental group practice. We were unable to obtain full-text articles for nine papers. The PRISMA flow diagram in figure 1 displays these results.

The majority of papers were from the USA (58\%), followed by Europe (19\%), and then Canada (15\%). There were only a handful of papers from elsewhere in the world $(7 \%)$. Papers frequently included more than one type of group practice. Family medicine was reported on most commonly $(76 \%)$, followed by surgical practices $(43 \%)$, and all others $(36 \%)$. Physicians $(94 \%)$ were the focus of almost all the papers rather than patients (26\%),

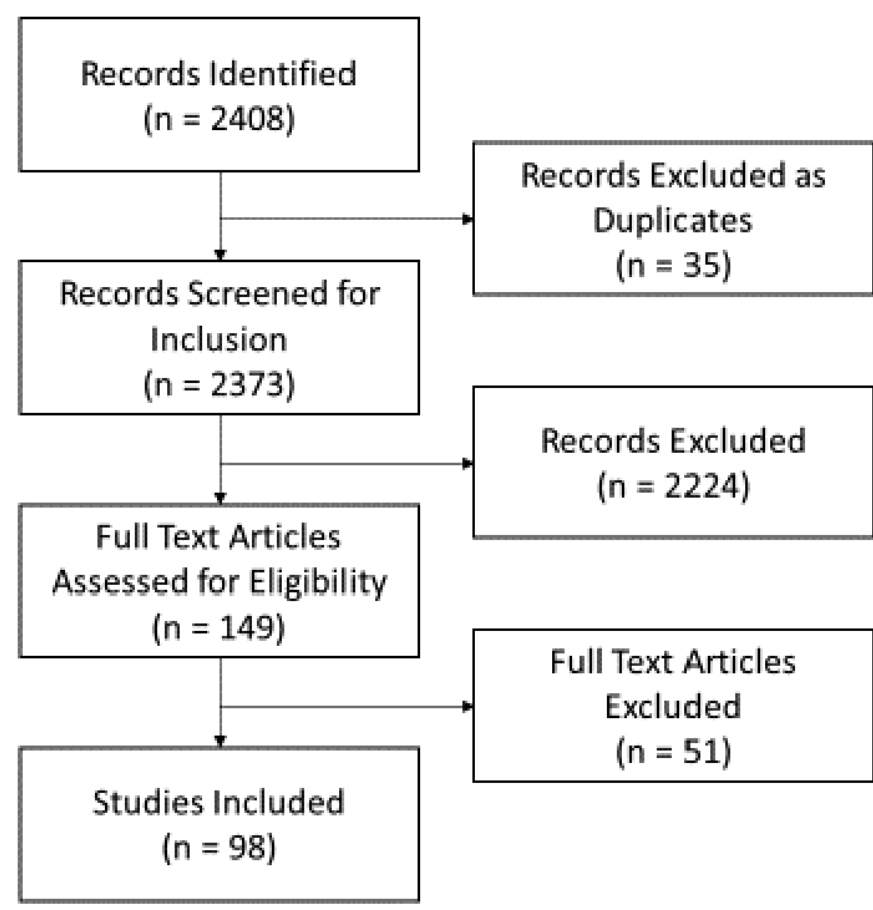

Figure 1 Preferred Reporting Items for Systematic Reviews and Meta-Analyses flow diagram for screened and included papers. allied health $(4 \%)$ or healthcare systems $(10 \%)$. Some papers touched on multiple populations. Most of the included papers were surveys $(63 \%)$. Group practices have been published on dating back until at least the 1960s. Recurring themes were evident over the years and are expanded on in the qualitative analysis. See table 1 for a full description of included papers.

\section{Group practices improve patient satisfaction and experience}

Sources that addressed patient outcomes are listed in online supplemental appendix B. Six of these provided evidence that group practices can result in improvements in patient satisfaction. ${ }^{23}{ }^{32-35}$ Four of these sources were surveys that assessed changes in satisfaction after the implementation or expansion of a group practice. This sense of satisfaction appeared to be most commonly due to better perceived access to care and

\begin{tabular}{|c|c|}
\hline Characteristic & n (\%) \\
\hline \multicolumn{2}{|l|}{ Region of study } \\
\hline USA & $56(57)$ \\
\hline Europe & $20(20)$ \\
\hline Canada & $16(16)$ \\
\hline Other & $7(7)$ \\
\hline \multicolumn{2}{|l|}{ Type of group practice } \\
\hline Family medicine or general practitioner & $74(76)$ \\
\hline Surgical & $42(43)$ \\
\hline Other & $35(36)$ \\
\hline \multicolumn{2}{|l|}{ Population studied } \\
\hline Physicians & $92(94)$ \\
\hline Patients & $25(26)$ \\
\hline Healthcare systems & $10(10)$ \\
\hline Allied health & $4(4)$ \\
\hline \multicolumn{2}{|l|}{ Type of publication } \\
\hline Survey & $62(63)$ \\
\hline Letter & $7(7)$ \\
\hline Case report & $6(6)$ \\
\hline Cohort study & $5(5)$ \\
\hline Abstract & $2(2)$ \\
\hline Case series & $2(2)$ \\
\hline $\mathrm{RCT}$ & $2(2)$ \\
\hline Systematic review & $2(2)$ \\
\hline Other & $10(10)$ \\
\hline \multicolumn{2}{|l|}{ Publications by decade } \\
\hline 1960-1969 & $6(6)$ \\
\hline 1970-1979 & $9(9)$ \\
\hline 1980-1989 & $5(5)$ \\
\hline 1990-1999 & $9(9)$ \\
\hline 2000-2009 & $23(23)$ \\
\hline 2010-present & $46(49)$ \\
\hline
\end{tabular}

$\mathrm{RCT}$, randomised controlled trial. 
quality of care. In contrast to this, one survey from 1975 identified a negative effect on patient satisfaction and experience. ${ }^{36}$ The Patient Perceptions of Integrated Care Survey identified that patients with multiple chronic diseases who perceive a higher level of integration within a group will use less emergency department and outpatient resources. ${ }^{37}$

\section{Patient quality of care}

The aspects of quality of care assessed by the papers included access to care, continuity of care, prescribing techniques, adherence of the physicians with established clinical guidelines, frequency of consultations, and unnecessary investigations and treatment. Twenty-two sources either identified an improvement in patient quality of care associated with group practices or a negative impact on patient quality of care. Two sources did not identify any differences in patient quality of care based on practice organisation. ${ }^{38} 39$

\section{Group practices improve patient quality of care}

Twenty-two sources demonstrated improvements in patient quality of care. This included objective measures with quality of care scores as well as patient perception as captured by surveys. Group practices were found to improve access to care, comprehensiveness, waiting times, time spent with patients, efficiency, patient safety and utilisation of resources according to patient-reported outcomes. ${ }^{6} 83340-48$ Patients perceived a higher quality of care with group practices with regard to tangibles (equipment and facilities), reliability, responsiveness, assurance and empathy. ${ }^{49}$ Physicians in group practices had higher quality of care scores, and adherence to guidelines was found to be better due to increased knowledge sharing and access to information. ${ }^{35} 445051$ Improvements in appropriate prescribing techniques were also associated with physicians working in group practices. ${ }^{7}{ }^{52}$ Physicians in group practices were also more likely to consult peers. ${ }^{53}$ More patient-centred medical home processes within a practice were associated with larger groups compared with solo and small group practices (1-2 physicians), although all types of practices have shown modest increases over time.$^{5455}$ However, a recent paper based on large surveys found no improvements in quality measures based on practice size. ${ }^{56}$

\section{Group practices negatively impact patient quality of care}

Six sources noted some negative impacts with group practices on patient quality of care. This included worse continuity of care and dilution of the patient-doctor relationship. ${ }^{41}{ }^{57}$ Group practices have also been found to order more investigations or treat inappropriately if there was a financial benefit. ${ }^{858} 59$ Additionally, a primary care internist who moved from a small practice to a large group practice after many years perceived that the level of care he was providing was compromised by the large group and payers setting targets for the group. ${ }^{60}$

\section{Physician quality of life, satisfaction and burnout}

Papers which assessed physician outcomes are listed in online supplemental appendix B. Twenty papers assessed the relationship between group practices and physician quality of life, satisfaction and burnout. Two papers did not find any significant difference in this area for group and solo practices. ${ }^{6162}$ Two main themes were identified from the sources including the following: group practices improve physician quality of life and satisfaction, and group practices lead to conflict and additional stress for physicians.

\section{Group practices improve physician quality of life and satisfaction}

Group practices were often found to improve the work-life balance and job satisfaction of physicians when compared with solo practices. ${ }^{9} 1963-67$ Being a member of a group practice led to less professional isolation, improved knowledge sharing and an improvement in professional development. ${ }^{9} 1968$ Improved attitudes about group practices in the Netherlands were related to an increased desire for contact and cooperation with other physicians ${ }^{69}$ Satisfaction with personal and lifestyle factors and optimism for the future was increased among physicians in group practices. ${ }^{70}$ Group practices were also associated with a decreased call burden and increased cross coverage of patients which directly impacts the quality of life for most physicians and their families. ${ }^{68}$ Due to the aforementioned benefits, group practices have also been noted to improve retention and recruitment initiatives, especially in rural or underserviced areas. ${ }^{6872}$

\section{Group practices lead to conflict and additional stress for physicians}

Seven sources have identified issues with group practices that create conflict and additional stress for physicians. These centre around the interpersonal relationships of the group members and sustainability. ${ }^{63}$ Poor interpersonal relationships lead to lower job satisfaction and a higher degree of professional burnout. ${ }^{73} 74$ Three papers identified that group practices were also associated with increased physician demands, decreased performance and reduced autonomy. ${ }^{74-76} \mathrm{~A}$ large survey of family physicians in Canada found that physicians in solo practice had more job satisfaction than those in group practices in a survey that was primarily assessing improved satisfaction with performing procedures. ${ }^{77}$

\section{Group practices improve physician competency}

Two papers addressed differences in physician competency. ${ }^{78} 79$ Family physicians and surgeons were found to be less likely to pass their respective maintenance of certification examinations if they were in a solo practice. This was thought to result from the ability to spend more time on quality improvement and education within a group practice.

\section{Facilitators and barriers associated with working in a group} practice

An important theme that arose during analysis was the identification of barriers and facilitators associated with 
forming or maintaining a group practice. These characteristics have direct impacts on patient care and physician quality of life, job satisfaction and burnout. Eight sources identified these characteristics and they are summarised in table $2 .^{10122280-84}$

\section{Group practices lead to higher incomes for physicians}

Seven sources from the USA, Taiwan and South Africa have identified increased individual earnings for physicians practising within group practices. ${ }^{15} 21$ 71 85-88 Physicians in group practices have also been shown to be more satisfied with their compensation. A letter published in 1968 highlighted income deferral by physicians until later in life when they were less productive as an additional benefit of group practices. ${ }^{89}$ Currently, larger groups may be forming in the USA as they are able to leverage insurers more effectively and build up more market share. ${ }^{3}$

\section{Costs of group practices are higher than solo practices}

Costs of group practices have been found to be higher than those of solo practices. ${ }^{20} 9091$ This may be due to more investment in technological costs that solo practices would not be able to afford. ${ }^{20} 91$ Some large group practices may also be more inefficient than solo or small group practices. ${ }^{92}$

\section{Group practices may improve the uptake of health information technology}

Physicians practising in groups are more likely to have greater access to health information technology (HIT) and were also more likely to correspond with their patients and other providers via email. ${ }^{793}$ Family physicians in the USA in solo practices were found to be less likely to adopt electronic health records when compared with those in group practices. ${ }^{9495}$ Data from two large surveys indicated a general trend towards increased use of HIT over time, but did not see a clear association between group size and an increased use of HIT. ${ }^{56}$

Table 2 List of barriers and facilitators for group practices

\begin{tabular}{|c|c|}
\hline Facilitators & Barriers \\
\hline Teamwork $^{8182}$ & Incompatible personalities ${ }^{1022}$ \\
\hline Leadership 2281 & Poor leadership ${ }^{12}$ \\
\hline Common vision $^{84}$ & $\begin{array}{l}\text { Different visions for the } \\
\text { group }^{12}\end{array}$ \\
\hline Patient-centred care ${ }^{81}$ & $\begin{array}{l}\text { Disagreements about } \\
\text { reimbursement }^{12}\end{array}$ \\
\hline Quality improvement ${ }^{81}$ & Legal and real estate issues ${ }^{10}$ \\
\hline Accountability $^{81}$ & Dissatisfied office staff ${ }^{12}$ \\
\hline Sense of ownership ${ }^{81}$ & $\begin{array}{l}\text { Fears about loss of } \\
\text { autonomy }\end{array}$ \\
\hline \multicolumn{2}{|l|}{ Sense of responsibility ${ }^{82}$} \\
\hline Cohesiveness $^{82} 83$ & \\
\hline
\end{tabular}

More physicians are practising in group practices and group practices are increasing in size

Many sources have tracked the rise in the number and type of group practices over the years (online supplemental appendix B). The definition of a group practice is very heterogeneous in the literature and previous work has been done in an attempt to classify groups. ${ }^{24}$ Often, groups of 1-19 physicians are classified as small or medium (further subclassified into groups of 1-2, 3-7, 8-12 and 13-19), and groups of 20 or more are classified as large (further subclassified into groups of 20-99 and $>100) .{ }^{54} 55$ The included papers show an increase in the absolute number of group practices and their sizes over the years.

In 1968, group practices were mostly limited to hospitals with most other physicians working in solo practices outside of the hospitals, and it was believed that group practices would not be taken up unless it was established as a desirable form of practice to society and healthcare professionals. $^{96} 97$ Now, in the USA, more physicians across all specialties are forming or joining larger groups, and groups of more than 100 physicians which usually have non-physician owners, have grown rapidly in recent years. $^{2-4}$ This increase has been driven by the benefits group practices can offer physicians. ${ }^{11} 1398-101$

Despite having a very different healthcare system, group practices have also grown in Canada. In 1970, 57\% of graduating physicians entered a group practice or partnership, $21 \%$ entered solo practice and $22 \%$ became salaried physicians. Surgeons and psychiatrists were most likely to enter solo practice. ${ }^{5}$ A survey of Canadian physicians in 1987 found that around half of the physicians were in either solo or group practices and the other half had some group practice arrangements for financial benefits. ${ }^{14}$ Government support was seen as a key factor in establishing group practices. ${ }^{14} 102$

\section{Group practices may help reduce costs within healthcare systems}

Group practices have the potential to impact healthcare systems financially, with respect to access to care, and appropriate utilisation of healthcare resources. Sources have shown that group practices of all sizes and most specialties have been shown to have more technical, cost and profit efficiencies than solo practices (online supplemental appendix B). ${ }^{103} 104$ This is thought to be due to the standardisation of processes. ${ }^{104}$ Group practices that focus on improved screening and monitoring may improve avoidable utilisation, cost and revenue. ${ }^{103}$ A higher level of integration perceived by patients with chronic illnesses also reduces utilisation of emergency department and outpatient resources. ${ }^{37}$ Income pooling within an obstetrical call group in a Canadian study led to decreased rates of elective induction of labour in a before and after study. ${ }^{105}$ Older data from the Physicians' Practice Cost and Income Survey in 1986 found no significant differences in practice efficiency between solo and group primary care practices in the USA. ${ }^{106}$ Additionally, 
a recent paper which included data from large surveys found that group size was not associated with an improvement in spending or quality. ${ }^{56}$

\section{DISCUSSION}

We were able to identify themes associated with the advantages and disadvantages that group practices have for patients, physicians and healthcare systems. It is important to note that the term 'group practice' refers to a broad range of practice types within the literature and there is no clear definition with respect to the critical pieces that define what a group practice is beyond the number of physicians and inclusion of one or more specialties. Organisations in the USA such as the America's Physician Groups and American Medical Group Association have been developed to represent physicians in various types of groups. Groups may be defined as single specialty with two or more physicians or multispecialty with any number of different specialties providing care to patients.

Themes involving patients included satisfaction and quality of care. Generally, patients seemed to be more satisfied with care that was being received from physicians in group practices. ${ }^{23} 323340$ From these studies, this appears to be due to increased access to care and decreased waiting times. Although continuity of care would seem to be a legitimate concern with a group practice as patients may be seeing different physicians on any given day, this was actually shown to be improved in one study. ${ }^{23}$ Concerns surrounding continuity of care were raised in one study which addressed non-adherent patients in a group practice. ${ }^{57}$ Furthermore, in a situation that is unique to a surgical group practice, patients did not seem to be concerned by the fact that they might not meet the surgeon who is operating on them until the day of their operation as they had confidence in any of the surgeons associated with the group. ${ }^{33}$

While it is important that patients are satisfied with the care they are receiving, it is imperative that they also receive high-quality care. Overall, most papers indicated that the quality of care increased with a group practice structure as measured objectively and subjectively. Adherence to guidelines and appropriate prescribing was better with group practices and quality of care scores improved. $^{75152}$ There were some notable exceptions including using radiation therapy for prostate cancer when it was not necessarily indicated because the group owned radiation facilities, and the increased use of laboratory investigations offered by the group. ${ }^{15} 5859$ This may have been driven by convenience as well as financial gain.

Overall, patients appear to benefit from group practices through improved quality of care, access and satisfaction. The data surrounding the impact of group practices on patients were presented in $24 \%$ of papers. This has been identified as an area for further research as we know that group practices are often formed to primarily benefit the physicians working within them. ${ }^{68} 72$
Numerous advantages of group practices for physicians have been identified from this scoping review. They include increased quality of life and satisfaction, decreased burnout, higher competency and financial gain. More attention has been paid to physician burnout in recent years as the prevalence is surprisingly high. ${ }^{17} 18$ Improving the quality of life and job satisfaction for physicians may help with this and group practices have the potential to help in these areas. Overall, most of the literature included in this review shows a positive association with group practices and physician quality of life and job satisfaction. These improvements result from a better work-life balance, shared call responsibilities, improved knowledge transfer, collaboration and decreased professional isolation. ${ }^{69366}$ Physicians in solo practices may still be able to pursue similar opportunities but may face logistical challenges due to isolation.

A notable area of decreased satisfaction results from poor interpersonal relationships. ${ }^{122}$ This can lead to the collapse of a group and highlights the need for group practice members to be compatible and share a common vision, especially if they are financially integrated. As groups become larger and larger, especially in the USA where groups of more than 100 physicians are not uncommon, relationships can become less collegial and autonomy may be lost. ${ }^{80}$ The importance of regular meetings with a shared sense of ownership and responsibility has been shown to be very important to group function and quality of care. ${ }^{81} 84$ Therefore, although groups have the potential to improve job satisfaction and quality of life for physicians, it depends on the overall functioning of the group and compatible personalities within the group for this to be achieved.

In the two papers assessing the level of physician competency (based on whether or not physicians were members of group practices) the overall impact seems to be positive with improved scores on certification examinations. ${ }^{7879}$ This is thought to be due to more knowledge transfer between group members and less professional isolation. The ability to approach and consult colleagues relatively easily about difficult or interesting clinical questions has the potential to enhance the learning of all group members and improve patient care.

Financially, group practices have been shown to improve incomes of physicians. This is most relevant in the USA where groups are often formed to gain negotiating leverage with payers. ${ }^{11} 13$ However, individual incomes also seem to be higher in other areas of the world such as New Zealand, South Africa and Taiwan. ${ }^{718687}$ The increased income may help offset costs associated with investments in equipment or technology that would not be feasible for solo physicians.

The impact of group practices on healthcare systems can be seen in improvements in access to care, system efficiencies, improved use of resources and adherence to guidelines. Some exceptions to this may include inappropriate use of resources if there is a financial gain. Moving forward, this will be an important area of study as there 
are many different healthcare systems in place around the world.

This scoping review has allowed us to identify gaps in the literature which can be addressed in the future. As demonstrated above, patient care is often not the focus of research into group practices. This needs to be addressed to ensure that we are improving the service that is being delivered to the end user, namely, the patient. Creating a shared or group practice is often beneficial to physicians, but if the patient experience or quality of care is negatively impacted, this needs to be understood. Additionally, it was difficult to separate different types of group practices in the literature. The definition of a group practice varies significantly and includes anywhere from 2 to $>100$ physicians and/or allied healthcare providers. ${ }^{24}$ This makes comparisons difficult. However, this scoping review has allowed us to perform a high-level overview of all types of group practices and in an attempt to identify all characteristics which are important to patients and physicians. The knowledge gaps we identified with respect to this issue includes a group practice definition and which elements contribute to a successful practice which benefits patients and physicians.

As part of the scoping review process, key stakeholders were consulted regarding this review. They included a patient advisor and members of other group practices. The patient advisor was included in the design of this study, verified the results and reviewed the final draft of this manuscript. Other group practice members verified the results by reviewing the themes and included references, ensuring that all relevant papers were included. The patient advisor and group practice members will help to guide further research in the future. Some of the authors of this paper are group practice members and will be using their practice for research that will focus on patient outcomes including quality of care and satisfaction, as well as physician outcomes including quality of life, satisfaction and burnout with guidance from this scoping review.

There are inherent limitations with a scoping review. This was meant to be a broad overview of the available literature and as such, the data are heterogeneous and do not lend itself well to a quality assessment. There may very well be a publication bias with this topic as authors may only be inclined to publish on group practices that have worked very well. We were unable to obtain the full text for nine papers. The included papers were also from many different regions and therefore, the conclusions may not be applicable to a particular country or region, however the objective of this review was to assess the advantages and disadvantages of group practices and common themes were identified that likely transcend many regional differences.

\section{CONCLUSION}

A group practice structure has many advantages for patients and physicians alike. Although the data are somewhat limited for patients compared with physicians, this scoping review has shown that there is a generally positive patient experience with some evidence of improved quality of care. There is also an increase in physician satisfaction and quality of life in groups that function well with compatible personalities. This scoping review has summarised the available literature based on our research question and has allowed us to identify two interesting areas of future investigation. First, it will be important to define exactly what the critical elements of a group practice are beyond the number of physicians as there is no standard definition that we were able to discern in this scoping review. This may then be used to guide the development of functional groups that are able to improve care and quality of life for both patients and providers. Second, although most of the available literature is directed towards the impact of group practices on physicians, addressing patient outcomes and perspectives is essential. This has been addressed in the literature more recently, and is an area which should be further developed.

Acknowledgements We would like to acknowledge Risa Shorr and Josée Skuce for their help in developing the search strategy and obtaining full-text articles for this scoping review.

Contributors HM, TZ, DIM and FB conceived the study. TZ and HM drafted the protocol and developed the research question. TZ and SA performed the title and abstract screening and full-text data extraction. TZ, HM, JB, TW and SR contributed to the thematic analysis and interpretation of the data. All authors read and approved the final manuscript. HM is the guarantor.

Funding The authors have not declared a specific grant for this research from any funding agency in the public, commercial or not-for-profit sectors.

Competing interests None declared.

Patient consent for publication Not required.

Provenance and peer review Not commissioned; externally peer reviewed.

Data availability statement All data relevant to the study are included in the article or uploaded as supplemental information. All data used are included in the article and supplemental appendices.

Supplemental material This content has been supplied by the author(s). It has not been vetted by BMJ Publishing Group Limited (BMJ) and may not have been peer-reviewed. Any opinions or recommendations discussed are solely those of the author(s) and are not endorsed by BMJ. BMJ disclaims all liability and responsibility arising from any reliance placed on the content. Where the content includes any translated material, BMJ does not warrant the accuracy and reliability of the translations (including but not limited to local regulations, clinical guidelines, terminology, drug names and drug dosages), and is not responsible for any error and/or omissions arising from translation and adaptation or otherwise.

Open access This is an open access article distributed in accordance with the Creative Commons Attribution Non Commercial (CC BY-NC 4.0) license, which permits others to distribute, remix, adapt, build upon this work non-commercially, and license their derivative works on different terms, provided the original work is properly cited, appropriate credit is given, any changes made indicated, and the use is non-commercial. See: http://creativecommons.org/licenses/by-nc/4.0/.

ORCID iD

Terry Zwiep http://orcid.org/0000-0003-4345-0215

\section{REFERENCES}

1 Fye WB. Presidential address: The origins and evolution of the Mayo Clinic from 1864 to 1939: a Minnesota family practice becomes an international "medical Mecca". Bull Hist Med 2010;84:323-57. 
2 Muhlestein DB, Smith NJ. Physician consolidation: rapid movement from small to large group practices, 2013-15. Health Aff 2016;35:1638-42.

3 Burns LR, Goldsmith JC, Sen A. Horizontal and vertical integration of physicians: a tale of two tails. Adv Health Care Manag 2013;15:39-117.

4 Liebhaber A, Grossman JM. Physicians moving to mid-sized, single-specialty practices. Track Rep 2007;18:1-5.

5 Paulick JM, Roos NP. The young physician: types of practice. Can Med Assoc J 1978;118:276-8.

6 Breon TA. Rural surgical practice: an lowa group model. Surg Clin North Am 2009;89:1359-66.

7 Damiani G, Silvestrini G, Federico B, et al. A systematic review on the effectiveness of group versus single-handed practice. Health Policy 2013;113:180-7.

8 Devlin RA, Hogg W, Zhong J, et al. Practice size, financial sharing and quality of care. BMC Health Serv Res 2013;13:446.

9 Feron J-M, Cerexhe F, Pestiaux D, et al. Gps working in solo practice: obstacles and motivations for working in a group? A qualitative study. Fam Pract 2003;20:167-72.

10 Hays RB, Sanderson L. Forming large group practices: is it worth it? Med J Aust 1994;161:494-6.

11 Casalino LP, Devers KJ, Lake TK, et al. Benefits of and barriers to large medical group practice in the United States. Arch Intern Med 2003;163:1958.

12 Crane HS, Dennis DA. Risks of a large group practice: a personal experience. Clin Orthop Relat Res 2003;407:67-70.

13 Casalino LP, Pham H, Bazzoli G. Growth of single-specialty medical groups. Health Aff 2004;23:82-90.

14 Williams AP, Vayda E, Stevenson HM, et al. A typology of medical practice organization in Canada. data from a national survey of physicians. Med Care 1990;28:995-1004.

15 Bailey RM. A comparison of internists in solo and fee-for-service group practice in the San Francisco Bay area. Bull N Y Acad Med 1968;44:1293-303.

16 Rothenberger DA, Burnout P. Physician burnout and well-being: a systematic review and framework for action. Dis Colon Rectum 2017;60:567-76.

17 West CP, Dyrbye LN, Erwin PJ, et al. Interventions to prevent and reduce physician burnout: a systematic review and meta-analysis. Lancet 2016;388:2272-81.

18 Shanafelt TD, Hasan O, Dyrbye LN, et al. Changes in burnout and satisfaction with work-life balance in physicians and the general us working population between 2011 and 2014. Mayo Clin Proc 2015;90:1600-13.

19 Romano M. United we do quite well, thank you. survey: physicians say practicing in groups is a better way to provide care. Mod Healthc 2001;31:34.

20 Heimeshoff M, Schreyögg J, Kwietniewski L. Cost and technical efficiency of physician practices: a stochastic frontier approach using panel data. Health Care Manag Sci 2014;17:150-61.

21 Kwietniewski L, Schreyögg J. Profit efficiency of physician practices: a stochastic frontier approach using panel data. Health Care Manag Sci 2018;21:76-86.

22 Rodríguez C, Pozzebon M. The implementation evaluation of primary care groups of practice: a focus on organizational identity. BMC Fam Pract 2010;11:15.

23 Tourigny A, Aubin M, Haggerty J, et al. Patients' perceptions of the quality of care after primary care reform: family medicine groups in Quebec. Can Fam Physician 2010;56:e273-82.

24 Kash B, Tan D. Physician group practice trends: a comprehensive review. J Hosp Med Manage 2016;2:1-8.

25 Arksey H, O'Malley L. Scoping studies: towards a methodological framework. Int J Soc Res Methodol 2005;8:19-32.

26 Levac D, Colquhoun H, O'Brien KK. Scoping studies: advancing the methodology. Implement Sci 2010;5:69.

27 Tricco AC, Lillie E, Zarin W, et al. PRISMA extension for scoping reviews (PRISMA-ScR): checklist and explanation. Ann Intern Med 2018;169:467-73.

28 Zwiep TM, Greenberg JA, Balaa F, et al. Impact of group practices on patients, physicians and healthcare systems: protocol for a scoping review. BMJ Open 2018;8:e022164.

29 McGowan J, Sampson M, Salzwedel DM, et al. PRESS Peer Review of Electronic Search Strategies: 2015 Guideline Statement. J Clin Epidemiol 2016;75:40-6.

30 Colorafi KJ, Evans B. Qualitative descriptive methods in health science research. HERD 2016;9:16-25.

31 IAP2. Core Values, Ethics, Spectrum - The 3 Pillars of Public Participation - InternationalAssociation for Public Participation. Available: https://www.iap2.org/page/pillars
32 Gawande SR, Benroth R. Comparison of patient satisfaction results during a period of rapid growth for a specialty group practice. Am $J$ Orthop 1999;28:236-8.

33 van den Heuvel B, Vair B, Porter G, et al. Patient compliance with a group model of care: the hernia clinic. Can J Surg 2012;55:259-63.

34 Cohidon C, Wild P, Senn N. Patient experience in primary care: association with patient, physician and practice characteristics in a fee-for-service system. Swiss Med Wkly 2018;148:w14601.

35 Ellis SD, Karim SA, Vukas RR, et al. Four needles in a haystack: a systematic review assessing quality of health care in specialty practice by practice type. Inquiry 2018;55:46958018787041.

36 Ritchey FJ. Solo and group physician practices, Family-Physician relationships and unmet critical health need in rural areas. San Antonio: Annual Meeting of the Southwestern Sociological Association, 1975.

37 Fryer A-K, Friedberg MW, Thompson RW, et al. Patient perceptions of integrated care and their relationship to utilization of emergency, inpatient and outpatient services. Healthc 2017;5:183-93.

38 Visca M, Donatini A, Gini R, et al. Group versus single handed primary care: a performance evaluation of the care delivered to chronic patients by Italian GPs. Health Policy 2013;113:188-98.

39 Roos NP. Impact of the organization of practice on quality of care and physician productivity. Med Care 1980;18:347-59.

40 Cohen DI, Breslau D, Porter DK, et al. Academic group practice. Med Care 1986;24:990-8.

41 Graham FE. Group versus solo practice: arguments and evidence. Inquiry 1972;9:49-60.

42 Gaal S, van den Hombergh P, Verstappen W, et al. Patient safety features are more present in larger primary care practices. Health Policy 2010;97:87-91.

43 Xierali IM. Physician multisite practicing: impact on access to care. J Am Board Fam Med 2018;31:260-9.

44 Stol DM, Hollander M, Nielen MMJ, et al. Implementation of selective prevention for cardiometabolic diseases; are Dutch general practices adequately prepared? Scand J Prim Health Care 2018;36:20-7

45 Bardos J, Loudon H, Rekawek P, et al. The association between solo versus group obstetrical practice model and delivery outcomes. Am J Perinatol 2019;36:907-10.

46 Ly DP, Glied SA. Variations in the service quality of medical practices. Am J Manag Care 2013;19:e378-85.

47 Orrantia E, works M. How to thrive in rural practice. Can Fam Physician 2005;51:1217-21.

48 Campbell JL, Ramsay J, Green J. Practice size: impact on consultation length, workload, and patient assessment of care. $\mathrm{Br} J$ Gen Pract 2001;51:644-50.

49 Lin H-C, Xirasagar S, Laditka JN. Patient perceptions of service quality in group versus solo practice clinics. Int J Qual Health Care 2004;16:437-45.

50 Perkins RB, Anderson BL, Gorin SS, et al. Challenges in cervical cancer prevention: a survey of U.S. obstetrician-gynecologists. Am J Prev Med 2013;45:175-81.

51 Ashworth M, Armstrong D. The relationship between general practice characteristics and quality of care: a national survey of quality indicators used in the UK quality and outcomes framework, 2004-5. BMC Fam Pract 2006;7:68.

52 Pichetti S, Sermet C, Godman B, et al. Multilevel analysis of the influence of patients' and general practitioners' characteristics on patented versus multiple-sourced statin prescribing in France. App/ Health Econ Health Policy 2013;11:205-18.

53 Kuyvenhoven MM, Pieters HM, Jacobs HM, et al. Do peer consultations improve quality of care in general practice? Qual Assur Heal care in J Int Soc Qual Assur Heal Care 1990;2:171-9.

54 Rittenhouse DR, Casalino LP, Shortell SM, et al. Small and mediumsize physician practices use few patient-centered medical home processes. Health Aff 2011;30:1575-84.

55 Wiley JA, Rittenhouse DR, Shortell SM, et al. Managing chronic illness: physician practices increased the use of care management and medical home processes. Health Aff 2015;34:78-86.

56 Baker LC, Pesko M, Ramsay P, et al. Are changes in medical group practice characteristics over time associated with Medicare spending and quality of care? Med Care Res Rev 2020;77:107755871881293.

57 Freemyer B, Stoff B. Ethical challenges with treating nonadherent patients in a group practice setting. J Am Acad Dermatol 2018;79:596-8.

58 Sellers EM. The influences of group and independent general practice on patient care: a comparative study in Ontario. Can Med Assoc J 1965;93:147-57. 
59 Hollenbeck BK, Kaufman SR, Yan P, et al. Urologist practice affiliation and intensity-modulated radiation therapy for prostate cancer in the elderly. Eur Urol 2018;73:491-8.

60 Landon BE. Use of quality indicators in patient care: a senior primary care physician trying to take good care of his patients. JAMA 2012;307:956-64.

61 Pasternak DP, Tuttle WC, Smith HL. Physician satisfaction in group practice: a comparison of primary care physicians with specialists. Ghaa J 1986;7:50-9.

62 Viehmann A, Kersting C, Thielmann A, et al. Prevalence of chronic stress in general practitioners and practice assistants: personal, practice and regional characteristics. PLoS One 2017;12:e0176658.

63 Koppula S, Brown JB, Jordan JM. Experiences of family physicians who practise primary care obstetrics in groups. J Obstet Gynaecol Can 2011;33:121-6.

64 Hueston WJ. Family physicians' satisfaction with practice. Arch Fam Med 1998;7:242-7.

65 Dowell AC, Hamilton S, McLeod DK. Job satisfaction, psychological morbidity and job stress among New Zealand general practitioners. N Z Med J 2000;113:269-72.

66 Streu R, Hawley S, Gay A, et al. Satisfaction with career choice among U.S. plastic surgeons: results from a national survey. Plast Reconstr Surg 2010;126:636-42.

67 Nørøxe KB, Pedersen AF, Bro F, et al. Mental well-being and job satisfaction among general practitioners: a nationwide crosssectional survey in Denmark. BMC Fam Pract 2018;19:130.

68 Connor RA, Hillson SD, Kralewski JE. An analysis of physician recruitment strategies in rural hospitals. Health Care Manage Rev 1995;20:7-18.

69 Verbeek-Heida PM. General practitioners' attitudes towards group practice in the Netherlands. Soc Sci Med 1969;3:249-58.

70 Stamps PL. Physicians and organizations: an uneasy alliance or a welcome relief? J Fam Pract 1995;41:27-32.

71 Moosa S, Luiz J, Carmichael T, et al. Insights of private general practitioners in group practice on the introduction of national health insurance in South Africa. Afr J Prim Health Care Fam Med 2016;8:e1-6.

72 Evashwick CJ. The role of group practice in the distribution of physicians in nonmetropolitan areas. Med Care 1976;14:808-23.

73 Streu R, Hansen J, Abrahamse P, et al. Professional burnout among US plastic surgeons: results of a national survey. Ann Plast Surg 2014;72:346-50.

74 Orton P, Orton C, Pereira Gray D. Depersonalised doctors: a crosssectional study of 564 doctors, 760 consultations and 1876 patient reports in UK general practice. BMJ Open 2012;2:e000274.

75 Mahoney AR. Factors affecting physicians' choice of group or independent practice. Inquiry 1973;10:9-18.

76 Mosaly PR, Mazur LM, Jones EL, et al. Quantifying the impact of cross coverage on physician's workload and performance in radiation oncology. Pract Radiat Oncol 2013;3:e179-86.

77 Rivet C, Ryan B, Stewart M. Hands on: is there an association between doing procedures and job satisfaction? Can Fam Physician 2007;53:93.

78 Schulte BM, Mannino DM, Royal KD, et al. Community size and organization of practice predict family physician recertification success. J Am Board Fam Med 2014;27:383-90.

79 Valentine MA, Barsade S, Edmondson AC, et al. Informa peer interaction and practice type as predictors of physician performance on maintenance of certification examinations. JAMA Surg 2014;149:597-603

80 Curoe A, Kralewski J, Kaissi A. Assessing the cultures of medical group practices. J Am Board Fam Pract 2003;16:394-8.

81 Solberg LI, Hroscikoski MC, Sperl-Hillen JM, et al. Transforming medical care: case study of an exemplary, small medical group. Ann Fam Med 2006;4:109-16.
82 Zazzali JL, Alexander JA, Shortell SM, et al. Organizational culture and physician satisfaction with dimensions of group practice. Health Serv Res 2007;42:1150-76.

83 Masselink LE, Lee S-YD, Konrad TR. Workplace relational factors and physicians' intention to withdraw from practice. Health Care Manage Rev 2008;33:178-87.

84 Suchman A, Mainali E, Hoffer Gittel J. Society of General Internal Medicine 34th Annual Meeting Abstracts of submissions. In: Addressing chronic conflict and problematic behaviors in a group practice, 2011.

85 Lowes R. Group practices pay better. Med Econ 2007;84:23-5.

86 Lin H-C, Chen C-S, Liu T-C, et al. Differences in practice income between solo and group practice physicians. Health Policy 2006;79:296-305.

87 McCormick IR, Thomson AN. What future for solo general practice? N Z Med J 1989;102:530-2.

88 Graham JW. Comparisons of solo practices and group practices. J Oral Surg 1979;37 Spec No A:A65-82.

89 Rose I. Aspects of group medical practice. Can Med Assoc J 1968;98:1118.

90 Davies S. General practice costs survey for financial year 1977/78. N Z Med J 1979;90:20-3.

91 Kwietniewski L, Heimeshoff M, Schreyögg J. Estimation of a physician practice cost function. Eur $\mathrm{J}$ Health Econ 2017;18:481-94.

92 Kimbell LJ, Lorant JH. Physician productivity and returns to scale. Health Serv Res 1977;12:367-79.

93 Mazurenko O, Hearld LR, Menachemi N. The relationship between the external environment and physician e-mail communication: the mediating role of health information technology availability. Health Care Manage Rev 2017;42:122-31.

94 Xierali IM, Phillips RL, Green LA, et al. Factors influencing family physician adoption of electronic health records (EHRs). J Am Board Fam Med 2013;26:388-93.

95 Rao SR, DesRoches CM, Donelan K, et al. Electronic health records in small physician practices: availability, use, and perceived benefits. J Am Med Informatics Assoc 2011;18:271-5.

96 Terris M. Introduction: origins and growth of group practice. Bull N Y Acad Med 1968;44:1277-81.

97 Weinerman ER. Problems and perspectives of group practice. Bull N Y Acad Med 1968;44:1423-34.

98 Sturm R. Effect of managed care and financing on practice constraints and career satisfaction in primary care. J Am Board Fam Pract 2002;15:367-77.

99 Sturm R. The impact of practice setting and financial incentives on career satisfaction and perceived practice limitations among surgeons. Am J Surg 2002;183:222-5.

100 Holden DM. Rural practice modes. Acad Med 1990;65:S32-40.

101 Schryver DL, Niederman GA, Johnson BA. Establishing a group practice "without walls". Health Care Strateg Manage 1993;11:18-21.

102 Wallace JD. Group practice: the way of the future? Can Med Assoc $J$ 1974:111:571.

103 Kralewski J, Dowd B, Knutson D, et al. The relationships of physician practice characteristics to quality of care and costs. Health Serv Res 2015;50:710-29.

104 Kwietniewski L, Schreyögg J. Efficiency of physician specialist groups. Health Care Manag Sci 2018;21:409-25.

105 Bland ES, Oppenheimer LW, Holmes P, et al. The effect of income pooling within a call group on rates of obstetric intervention. CMAJ 2001;164:337-9.

106 Defelice LC, Bradford WD. Relative inefficiencies in production between solo and group practice physicians. Health Econ 1997;6:455-65. 